\title{
The efficacy of a custom-fabricated nasal mask on gas exchange during nasal intermittent positive pressure ventilation
}

\author{
T. Tsuboi, M. Ohi, H. Kita, N. Otsuka, H. Hirata, T. Noguchi, K. Chin, M. Mishima, K. Kuno
}

\begin{abstract}
The efficacy of a custom-fabricated nasal mask on gas exchange during nasal intermittent positive pressure ventilation. T. Tsuboi, M. Ohi, H. Kita, N. Otsuka, H. Hirata, T. Noguchi, K. Chin, M. Mishima, K. Kuno. C) ERS Journals Ltd 1999.

ABSTRACT: Commercially available nasal masks have a large mask volume and give rise to considerable air leaks around the mask during nasal intermittent positive pressure ventilation (NIPPV) which may reduce alveolar ventilation ( $V$ A per breath).

The effects of a custom-fabricated nasal mask (F-mask) versus a commercially available mask (C-mask) on arterial blood gas measurements, dead space including both physiological and apparatus dead space $\left(V_{D}\right)$, air leak and $V_{\mathrm{A}}$ per breath were compared in patients with restrictive thoracic disease during short-term NIPPV sessions while using a volume cycled ventilator with equivalent settings for both masks.

The mask volume of the C-mask was significantly larger than that of the F-mask $(\mathrm{p}<0.003)$. The arterial carbon dioxide tension $\left(\mathrm{Pa}_{\mathrm{a}} \mathrm{CO}_{2}\right)$ during NIPPV with either the F-mask $(5.56 \pm 1.35 \mathrm{kPa})($ mean \pm sD) or the $\mathrm{C}$-mask $(6.87 \pm 0.96 \mathrm{kPa})$ was significantly lower than during spontaneous breathing $(7.75 \pm 0.81 \mathrm{kPa} ; \mathrm{p}<0.003)$, but the $\mathrm{Pa}_{3}, \mathrm{CO}_{2}$ decreased more during NIPPV with the F-mask than with the $C$-mask $(p<0.003)$. The $V D$ was significantly smaller $(p<0.03)$, the air leak was significantly less $(p<0.03)$, and the $V_{A}$ per breath was significantly larger $(p<0.03)$ during NIPPV with the F-mask than with the $\mathrm{C}$-mask.

In conclusion, nasal intermittent positive pressure ventilation with the F-mask was more effective than nasal intermittent positive pressure ventilation with the commercially available mask due to its smaller dead space and less air leak. Further studies are needed to extend these results to all the commercially available-masks.
\end{abstract}

Eur Respir J 1999; 13: 152-156. \author{
Kyoto, Japan \\ Correspondence: M. Ohi \\ Dept of Clinical Physiology \\ Chest Disease \\ Research Institute \\ Kyoto University \\ 53 Shogoin Kawahara-cho \\ Sakyoku \\ Kyoto 606 \\ Japan \\ Fax: 81757513854
}

Dept of Clinical Physiology, Chest Disease Research Institute, Kyoto University,

Keywords: Air leakage around a mask commercially available nasal mask custom-fabricated nasal mask dead space ventilation

nasal intermittent positive pressure ventilation

restrictive thoracic disease

Received: August 251997

Accepted after revision September 151998
Nasal intermittent positive pressure ventilation (NIPPV) is used worldwide for domiciliary treatment of chronic ventilatory disorders [1]. This can be attributed to the fact that commercially available masks (C-masks) for nasal continuous positive airway pressure (CPAP) commonly used to treat obstructive sleep apnoea are also useful for NIPPV [1]. However, C-masks have a large mask volume $(V \mathrm{~m})[2]$, and presumably lead to increased $\mathrm{CO}_{2}$ rebreathing. Custom-fabricated nasal masks (F-masks) have been reported to provide a better fit and a greater interface seal than C-masks [3]. Therefore, we hypothesized that NIPPV with a F-mask with an extremely small $V \mathrm{~m}$ and less air leakage around the mask (ALm) may increase alveolar ventilation, and thus improve arterial blood gases more effectively than a C-mask.

\section{Materials and methods}

\section{Study subjects}

Twelve (six male and six female) patients with restrictive thoracic disease (RTD) participated in this study; nine had pulmonary tuberculosis sequelae and three had kyphoscoliosis. The mean \pm SD values for the patients were age $60 \pm 11$ yrs, height $152 \pm 18 \mathrm{~cm}$, weight $39 \pm 9 \mathrm{~kg}$, vital ca- pacity (VC) $0.95 \pm 0.27 \mathrm{~L}$, percentage of predicted $\mathrm{VC}$ $32 \pm 6 \%$. All patients had experienced NIPPV using the Cand F-masks. They were in a stable state, and gave their written informed consent. The experimental protocol was approved by the ethics committee of our institute.

\section{Methods}

NIPPV apparatus. Positive pressure breath were delivered via a nasal mask connected through a hot-wire respiratory flowmeter (RF-H; Minato Medical Science Co., Osaka, Japan) to a volume-cycled home ventilator (Companion 2800; Puritan-Bennett Int., Middlesex, UK) in a controlled mechanical ventilation mode (CMV). Mean \pm SD settings of the ventilator were as follows; tidal volume $0.53 \pm 0.13 \mathrm{~L}$, respiratory rate $27 \pm 4$ breaths $\cdot \mathrm{min}^{-1}$ and ratio of inspiratory to expiratory time $0.75 \pm 0.28$. In two patients, supplemental oxygen was delivered through a side port in the ventilator, and was mixed with the inspired air (calculated inspired oxygen fraction was 0.30 ).

Masks for NIPPV. A C-mask (nasal CPAP mask, mid-size; Healthdyne Technologies, Marietta, GA, USA) with the mask hole closed and an F-mask were used. The F-mask was custom-made for individual patients as described by McDermott et al. [3]. The procedure for making Fmasks in our institute is as follows: 1) The patients must 
be in supine position with their eyes sealed and with their nose plugged, and breathe air or supplemental oxygen through a mouthpiece (fig. 1a). 2) A moulage impression is made of irreversible alginate impression material (Aroma fine DF 2 Normal set; GC Co., Tokyo, Japan) backed with orthopaedic plaster gauze (fig. 1b). 3) The impression is poured in dental stone, and the interface is outlined in pencil on the moulage cast (fig. 1c). 4) The F-mask is created using resin (Ostron 2; GC Co.) on the moulage cast. A connector (Swivel connector (double); Gibeck Respiration AB, Vasby, Sweden) for the ventilator tube is attached to the interface. Four attachment parts made of resin are added to the lateral phalanges for attachment of straps. Finally, after the inner surface of the interface is coated with dental softtissue conditioner (GC Soft liner; GC Co.), the interface is pressed to the patient's face (fig. 1d).

With the C-mask, commercially available headgear (Headgear; Healthdyne Technologies) was used (fig. 2a).
With the F-mask, commercially available straps (Portex Tracheostomy Tube Holder; Portex Ltd., Hythe, UK) and/ or wide contractile bandage were used (fig. 2b).

The $V \mathrm{~m}$ was measured by filling the mask with water and using the patient's facial moulage cast to displace water corresponding to the nasal volume.

\section{Experimental protocols and measurements}

Arterial blood was sampled for baseline measurement of blood gases in an arterial blood gas analyser (ABL300; Radiometer, Copenhagen, Denmark) while ten patients breathed room air, and two received supplemental oxygen at $2 \mathrm{~L} \cdot \mathrm{min}^{-1}$ via nasal prongs. All the patients were then subjected to two NIPPV sessions using the same ventilator settings and the different masks in random order on the same day. All patients were instructed to stay awake and to keep their mouths closed during each NIPPV session. Throughout each session, the following parameters were



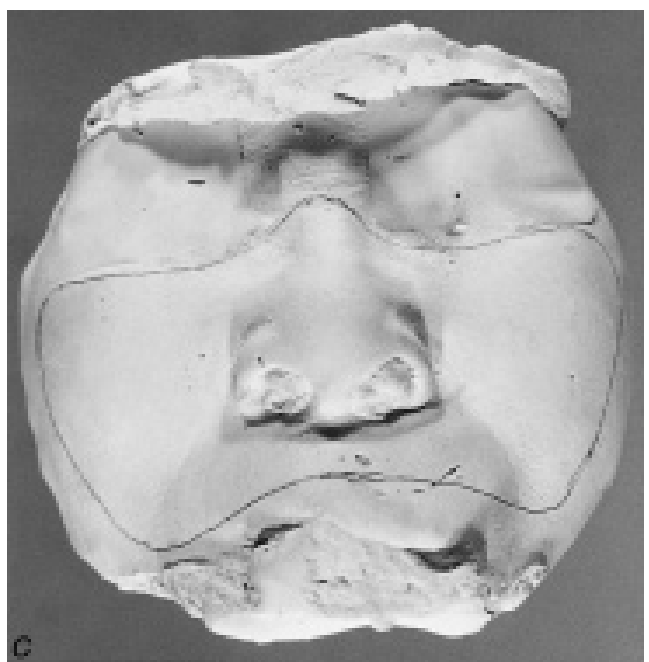

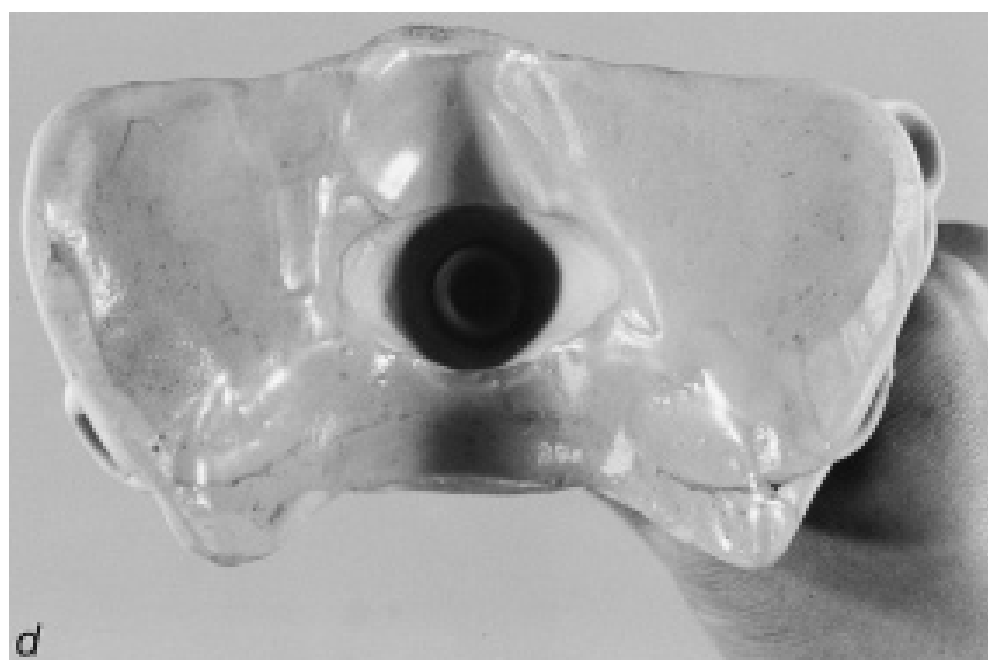

Fig. 1. - a) Patient breathing through mouthpiece with eyes sealed and with nose plugged; b) a moulage impression made of irreversible alginate impression material backed with orthopedic plaster gauze; c) nasal interface outlined on the moulage cast; d) inner surface of the custom-fabricated nasal mask coated with soft liner. 

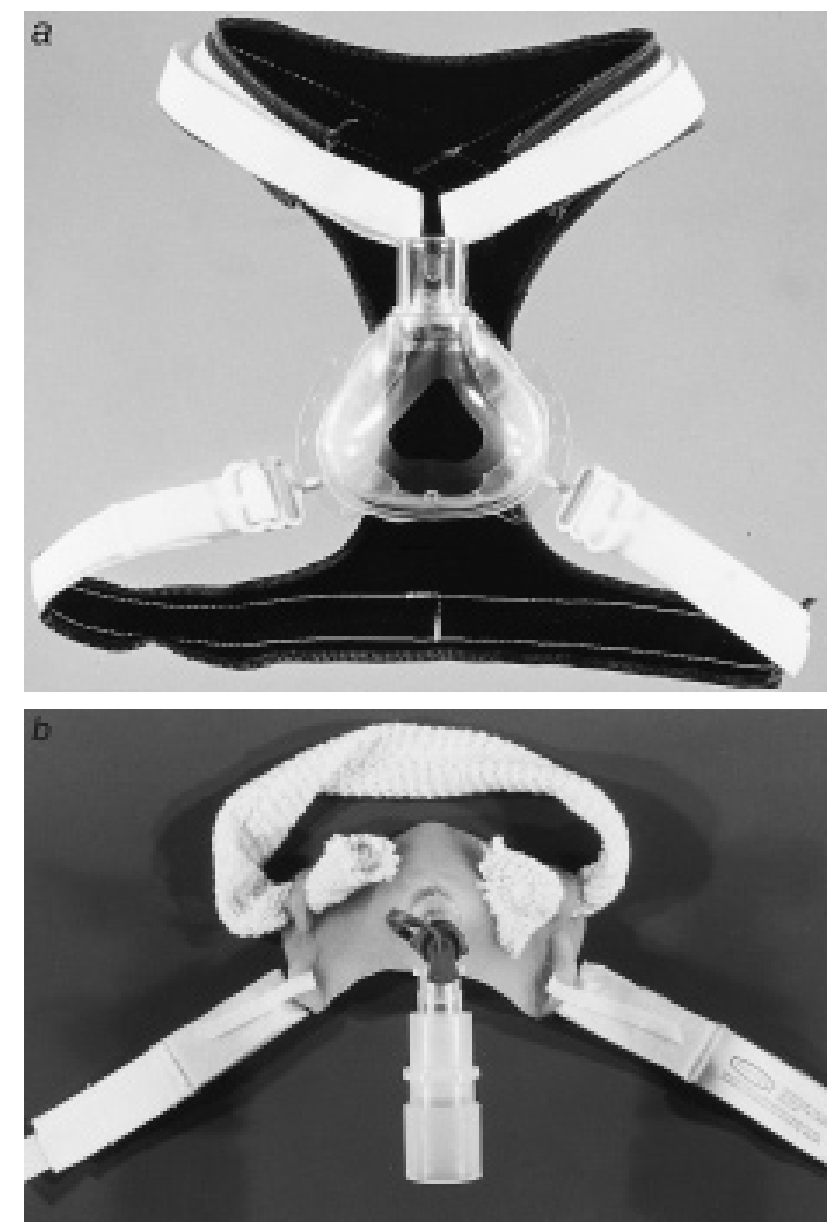

Fig. 2. - a) Commercially available nasal mask with headgear; b) custom-fabricated nasal mask with commercially available straps and wide contractile bandage.

monitored continuously: transcutaneous carbon dioxide tension $\left(P \mathrm{tc}, \mathrm{CO}_{2}\right)$ with a transcutaneous electrode (Cutaneous $\mathrm{PO}_{2} / \mathrm{PCO}_{2}$ Monitor 9000; Kontron Ltd., Switzerland), inspiratory $(V \mathrm{TI})$ and expiratory tidal volumes $(V \mathrm{TE})$ with a hot-wire respiratory flowmeter (RF-H) connected to a volume integrator (RM 300; Minato Medical Science Co.), and mask pressure $(P \mathrm{~m})$ with a differential pressure transducer(TP603T, $+50 \mathrm{cmH}_{2} \mathrm{O}$; Nihon-Kohden, Tokyo, Japan). Each session was performed for $>30 \mathrm{~min}$ and terminated 5 min after the $P \mathrm{tc}, \mathrm{CO}_{2}$ readings became stabilized. During the last 5 min of each session, the mean \pm sD peak $P$ m, and ALm per breath were calculated. At the end of each session, arterial blood was sampled to measure blood gases.

In six patients, the inspired and expired $\mathrm{CO}_{2}$ concentrations were measured in a mass spectrometer (MGA2000; Airspec, Kenton, UK). During the last $5 \mathrm{~min}$ of each session, the mean \pm SD of ALm, physiological and apparatus dead space $(V \mathrm{D})$ per breath, and alveolar ventilation $(V \mathrm{~A})$ per breath were calculated.

\section{Data analysis}

ALm was calculated as the difference between the $V$ TI and the $V$ TE. Assuming that there were no air leaks around the masks and through the mouth during the expiratory phase and hence the true tidal volume was equivalent to the $V \mathrm{TE}$, and that $\mathrm{CO}_{2}$ leakage around the mask during the inspiratory phase was negligible, then the $V \mathrm{D}$ was calculated from the mean inspiratory carbon dioxide fraction $\left(F \mathrm{I}, \mathrm{CO}_{2}\right)$ and expiratory carbon dioxide fraction $\left(F \mathrm{E}, \mathrm{CO}_{2}\right)$, and also from the fractional alveolar $\mathrm{CO}_{2}$ concentration $\left(F \mathrm{~A}, \mathrm{CO}_{2}\right)$ by the following formula:

$$
V \mathrm{D}=V \mathrm{TE}\left(F \mathrm{~A}, \mathrm{CO}_{2}-F \mathrm{E}, \mathrm{CO}_{2}\right) /\left(F \mathrm{~A}, \mathrm{CO}_{2}-F \mathrm{I}_{2} \mathrm{CO}_{2}\right)
$$

where the $F \mathrm{~A}, \mathrm{CO}_{2}$ was calculated from the arterial carbon dioxide tension $\left(\mathrm{Pa}_{\mathrm{a}} \mathrm{CO}_{2}\right)$ data as follows:

$$
F \mathrm{~A}, \mathrm{CO}_{2}=P \mathrm{a}, \mathrm{CO}_{2}(\mathrm{kPa}) / 95.1(\mathrm{kPa})
$$

The $V$ A per breath was then calculated by the following formula:

$$
V \mathrm{~A}=V \mathrm{TE}(1-V \mathrm{D} / V \mathrm{TE})
$$

\section{Statistical analysis}

The results were expressed as the mean \pm SD. For statistical comparisons, the Wilcoxon signed-rank test and the Friedman test were used. A p-value $<0.05$ was considered significant.

\section{Results}

All the patients preferred the F-mask. The average $V \mathrm{~m}$ of the F-mask was $8 \pm 2 \mathrm{~mL}$, whereas the $V \mathrm{~m}$ of the C-mask was $133 \pm 5 \mathrm{~mL}$. The mean $\pm \mathrm{SD} P \mathrm{a}_{1} \mathrm{CO}_{2}$ during NIPPV with either of the masks was significantly lower than during spontaneous breathing $(7.75 \pm 0.81 \mathrm{kPa})$, but $P \mathrm{a}, \mathrm{CO}_{2}$ decreased more significantly when the F-mask $(5.56 \pm 1.35$ $\mathrm{kPa})$ rather than the C-mask $(6.87 \pm 0.96 \mathrm{kPa})$ was used (fig. 3). The ALm was significantly lower when the F-mask $\left(43 \pm 34 \mathrm{~mL} \cdot\right.$ breath $\left.^{-1}\right)$ rather than the C-mask $(90 \pm 52 \mathrm{~mL}$. breath $^{-1}, \mathrm{p}<0.003$ ) was used. The peak $\mathrm{Pm}$ with the F-mask $\left(30 \pm 5 \mathrm{cmH}_{2} \mathrm{O}\right)$ was significantly higher than that with the $\mathrm{C}$-mask $\left(24 \pm 4 \mathrm{cmH}_{2} \mathrm{O}, \mathrm{p}<0.003\right)$. In the six patients in whom $\mathrm{FI}, \mathrm{CO}_{2}$ and $\mathrm{FE}, \mathrm{CO}_{2}$ were measured, ALm per breath was significantly less, $V \mathrm{D}$ per breath was significantly smaller, $V$ A per breath was significantly larger and $P \mathrm{a}, \mathrm{CO}_{2}$ was significantly lower during NIPPV using the F-mask (table 1).

\section{Discussion}

In this study, the values of arterial blood gases improved much more after NIPPV using the F-mask rather than the C-mask. The $V \mathrm{~m}$ of the F-mask was significantly less than that of the $\mathrm{C}$-mask; the $V \mathrm{D}$ per breath was significantly lower, and the $V \mathrm{~A}$ per breath was significantly larger. Moreover the F-mask was found to support a higher mask pressure with fewer interface leaks.

Reduction in $V \mathrm{D}$ ventilation during noninvasive positive pressure ventilation has been widely recommended [2]. In the present study, it was demonstrated that, in case of interfaces with smaller internal volume, $V \mathrm{D}$ ventilation was much less. 



Fig. 3. - Values of arterial blood gases while breathing spontaneously (Spontaneous), while receiving nasal intermittent positive pressure ventilation (NIPPV) using the commercially available nasal mask (Cmask), and while receiving NIPPV using the custom-fabricated nasal mask (F-mask). $P \mathrm{a}, \mathrm{O}_{2}$ : arterial oxygen tension; $\mathrm{Pa}_{\mathrm{a}} \mathrm{CO}_{2}$ : arterial carbon dioxide tension. - - - : mean. The values of blood gases were significantly improved during NIPPV using either mask than during spontaneous breathing, and significantly better when the F-mask rather than the C-mask was used. *: $\mathrm{p}<0.05$, versus spontaneous breathing; ${ }^{+}: \mathrm{p}<0.05$ versus the $\mathrm{C}$-mask.

It has been reported that air leakage around the interface is reduced more effectively when using custom-fabricated interfaces than when using commercially available masks [3]. The air leakage was quantified and it was found in Fmasks that ALm was much less than in that of C-masks. When using pressure-preset ventilators with the ability to compensate air leaks such as bilevel positive airway pressure (BiPAP) (Respironics Inc., Murrysville, PA, USA),
ALm may have less influence on gas exchange. However, a previous study has reported that some patients were unable to tolerate noninvasive positive pressure ventilation due to ALm and that a new full face mask minimizing air leaks improved patients' tolerance to NIPPV [4].

Previous studies have suggested that the resetting of the respiratory sensitivity to $\mathrm{CO}_{2}$ may be the most important mechanism resulting in an improvement in daytime blood gases during spontaneous breathing in patients receiving nocturnal ventilatory support [1]. This hypothesis was supported by a recent study [5] demonstrating that patients who showed the greatest decrease in mean overnight $P \mathrm{tc}, \mathrm{CO}_{2}$ while receiving nocturnal ventilation also showed the greatest decrease in resting daytime $\mathrm{Pa}_{\mathrm{a}} \mathrm{CO}_{2}$. In the present study, the decrease of $P \mathrm{a}_{1} \mathrm{CO}_{2}$ was more significant when the F-mask rather than the $\mathrm{C}$-mask was used, indicating that $\mathrm{Pa}_{\mathrm{a}} \mathrm{CO}_{2}$ during spontaneous breathing may decrease to a greater extent when using the F-masks.

In patients with severe restrictive thoracic disease like those in the present study, a high level of inspiratory pressure is necessary to provide an adequate tidal volume to reduce $P \mathrm{a}, \mathrm{CO}_{2}$. On the other hand, in patients with less severe restrictive defects or with obstructive disorders, ventilation may be effectively improved during NIPPV using a lower level of inspiratory pressure. ALm under a lower level of inspiratory pressure seems to be less than that under higher level. Therefore, these results with regard to ALm could not be systematically applied in other situations. Nonetheless it is believed that even in patients with mild ventilatory defects, when using interfaces with less internal volume, $\mathrm{CO}_{2}$ rebreathing could be reduced and hence inspiratory pressure and/or tidal volume in the ventilator settings could be decreased further, which might improve patients' tolerance to noninvasive ventilation.

All patients participating in the present study used a medium sized C-mask such that the difference in $V \mathrm{~m}$ between the C-and F-mask would be almost the same value. However, practically, the C-mask should be selected to minimize ALm. In patients for whom a smaller sized Cmask is more suitable than a medium sized C-mask, the difference in $\mathrm{ALm}, V \mathrm{D}$, and $V \mathrm{~A}$ per breath, and arterial blood gases between the F-mask and the smaller sized Cmask would be less than that obtained in the present study.

A C-mask made by only one industrial company was used in our tests. There appear to be other C-masks which more effectively reduce ALm. Therefore, further studies are needed to extend our results to all C-masks. However, trials comparing more than two types of masks could not be performed on the same day since the majority of our patients could not continue daytime NIPPV for $>2 \mathrm{~h}$ due to their abdominal distention and urge to urinate.

The entire mask-making procedure took a considerable amount of time, approximately $3 \mathrm{~h}$, and it cost approximately US\$30 when a trained doctor made an F-mask. The materials cost approximately US $\$ 40$ for the first mask. The F-mask could be used for approximately 18 months without any repairs. Repair of the F-mask took $\sim 20$ min and the materials cost approximately US\$20. On the other hand, the masks commercially available in Japan cost approximately US\$140-170, and can be used for approximately 8 months in our experience. Therefore the F-mask seems to be more economical compared with the C-mask.

In conclusion, when using volume cycled ventilators in controlled mechanical ventilation in patients with 
Table 1. - Comparison between the two masks for air leakage around the mask per breath (ALm), dead Space (VD) including the physiological and apparatus VD per breath, alveolar ventilation (VA per breath) and arterial carbon dioxide tension $\left(\mathrm{Pa}, \mathrm{CO}_{2}\right)$ in six patients

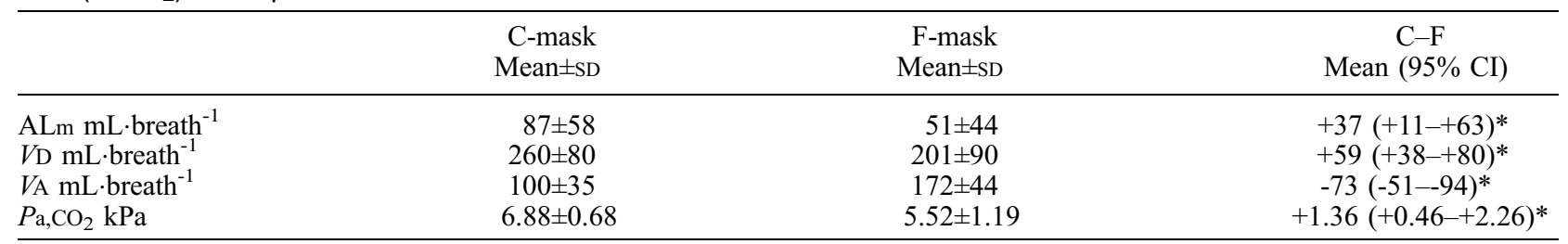

C: commercially available nasal mask; F: custom-fabricated nasal mask; C-F: difference between the C- and F-masks; CI: confidence interval. *: $\mathrm{p}<0.03$.

restrictive thoracic disease, nasal intermittent positive pressure ventilation proved to be more effective when a custom-fabricated nasal mask rather than a commercially available mask was used. This was probably due to less dead space ventilation and to fewer air leaks around the custom-fabricated nasal mask. Further studies are needed to extend these results to all commercially available mask(s), because only a one size commercially available mask made by one industrial company was tested in this study. In any case, interfaces with less internal volume and fewer air leaks should be used for nasal intermittent positive pressure ventilation.

\footnotetext{
Acknowledgements. The authors thank Y. Tsuboi, Dept of Oral and Maxillofacial Surgery, Faculty of Medicine, Kyoto University, for making the custom-fabricated nasal masks and for teaching us how to make them.
}

\section{References}

1. Hill NS. Noninvasive ventilation: Does it work, for whom, and how? Am Rev Respir Dis 1993; 147: 10501055.

2. CornetteA, Chabot F, Mougel D, Bouderhem D, Delorme N, Polu JM. Failure of nasal intermittent positive pressure ventilation due to excessive dead space of the mask and connecting tubing. Am J Respir Crit Care Med 1995; 151: A435.

3. McDermott I, Bach JR, Parker C, Sortor S. Customfabricated interfaces for intermittent positive pressure ventilation. Int J Prosthodont 1989; 2: 224-233.

4. Criner GJ, Travaline JM, Brennan KJ, Kreimer DT. Efficacy of a new full face mask for noninvasive positive pressure ventilation. Chest 1994; 106: 1109-1115.

5. Meecham-Jones DJ, Paul EA, Jones PW, Wedzicha JA. Nasal pressure support ventilation plus oxygen compared with oxygen therapy alone in hypercapnic COPD. Am J Respir Crit Care Med 1995; 152: 538-544. 